\title{
Quality Evaluation of Fritillaria thunbergii by High-Performance Liquid Chromatography Fingerprinting Coupled with Multicomponent Quantitative Analysis
}

\author{
Ruoxu $\mathrm{He}^{1}$, Qing $\mathrm{Ma}^{2}$, Yin $\mathrm{Lu}^{2^{*}}$ and Zhong Jiang ${ }^{1^{*}}$ \\ ${ }^{1}$ First Clinical Medical College, Zhejiang Chinese Medical University, Hangzhou 310006, China \\ ${ }^{2}$ College of Biology and Environmental Engineering, Zhejiang Shuren University, Hangzhou 310015, China \\ *For correspondence: uyin_zjsru@aliyun.com; jiangz_tcm@aliyun.com \\ Received 15 August 2020; Accepted 10 September 2020; Published 10 December 2020
}

\begin{abstract}
Medicinal Zhebeimu refers to the dried bulb of the plant Fritillaria thunbergii, which belongs to the family Liliaceae. Their main production sites are in Zhejiang, Jiangsu, and Anhui Provinces, with alkaloids as the main components. In this study, according to Chinese Pharmacopoeia, different mobile phases were investigated. Because most alkaloids in Fritillaria do not have UV absorption, evaporative light scattering detection (ELSD) was used instead of UV to produce the HPLC-ELSD spectra for five $F$. thunbergii samples and other samples, e.g. F. chuanxiensis, F. anhuiensis, and $F$. ussuriensis. Comparison showed significant differences exist among these species. There was no significant difference between the $F$. thunbergii samples produced in Zhejiang, which clustered together in both the cluster analyses. However, the $F$. thunbergii samples produced in Nantong, Jiangsu and the F. thunbergii samples produced in Zhejiang were separated in the clustering chart, which may be caused by different cultivation environments. The obtained results showed that the combination of quantitative analysis and chromatographic fingerprint analysis can be readily utilized for quality control purposes, offering a comprehensive strategy for quality evaluation of $F$. thunbergii and related products. (C) 2021 Friends Science Publishers
\end{abstract}

Keywords: Authenticity analysis; Fingerprint; Fritillaria thunbergii; HPLC; Active ingredients; China

\section{Introduction}

Chinese medicinal materials are the material basis for the application of traditional Chinese medicine (TCM) in disease prevention and treatment. Their quality directly affects the clinical efficacy of TCM, the experimental research of Chinese herbal medicines, and the quality control of Chinese patent medicines. There are many kinds of Chinese Herbal Medicine (CHM) in China with a wide range of sources, and the treatment by using these medicines vary from place to place. In the market, fake products are often claimed to be genuine, and so-called "superior" products are of a low quality. Such products include not only certified products but also substitutes, fake products, and adulterants with a great discrepancy in quality.

The active ingredients in CHM are mainly secondary metabolites from plants (or animals). These ingredients are influenced by factors, such as the place of production, climate, ecological environment, cultivation or culturing techniques and conditions, harvest seasons, storage conditions, etc., significant differences also occur in the secondary metabolites from the same plant or animal species (Liu et al. 2016). In addition, most Chinese medicinal materials need to be processed and treated before being used as medicine. Different processing and treatment methods also influence the composition of CHM, leading to unstable chemical compositions and quality, which in turn affects their clinical efficacy (Gao et al. 2011). Therefore, it is particularly important to find a method that can comprehensively and truly evaluate the quality of CHM.

With an in-depth study on the chemical composition of TCM and the technical improvement of modern instrumental analysis, methods of identification and quality evaluation of TCM are no longer limited to traditional and classic identification measures, such as morphological and microscopic identification. Various modern instruments have been used to perform physical and chemical identifications to reveal the inherent quality of TCM $(\mathrm{Qu}$ et al. 2014). However, owing to the long-term influence of the quality control mode for chemicals, quality of Chinese medicinal materials is also monitored by measuring the content of an effective or an active ingredient to determine the quality of specific medicinal materials. This mode does not fulfill the requirements of the overall efficacy that TCM advocates and cannot provide a comprehensive or reasonable evaluation of Chinese medicinal materials.

To cite this paper: He R, Q Ma, Y Lu, Z Jiang (2021). Quality evaluation of Fritillaria thunbergii by high-performance liquid chromatography fingerprinting coupled with multicomponent quantitative analysis. Intl J Agric Biol 25:131-138 
Compound recipes are a significant feature of CHM.

The chemical composition of a single herbal medicine is already quite complex with more than one active ingredient. Sometimes, synergistic effect between the components has a therapeutic role, and often, the antagonistic effect is curative. Therefore, a comprehensive quality evaluation of Chinese medicinal materials should not solely rely on a quantitative determination or qualitative identification of single or several chemical components (Sun et al. 2013). The chromatographic fingerprints of Chinese herbal medicines can provide comprehensive information about their quality and reveal most or even all of the effective components in herbal medicines or all of the active ingredients. This approach is more comprehensive and typical than the detection of the contents of a single component. Combined with other measures, it can be used to determine the quality and authenticity of Chinese medicinal materials.

Fritillaria refers to the bulb of many plants that belong to the genus Fritillaria L. in the family Liliaceae. Chinese Pharmacopoeia (2020) includes nine species of Fritillaria, which are $F$. thunbergii, $F$. cirrhosa, $F$. unibracteata, $F$. przewalskii, $F$. delavayi, $F$. ussuriensis, $F$. pallidiflora, $F$. walujewii and $F$. hupehensis. Among these species, the original plants of the medicinal material of $F$. chuanxiensis have been determined to be $F$. cirrhosa, $F$. unibracteata, $F$. przewalskii or $F$. delavayi; and the original plants of Siberian fritillary are $F$. pallidiflora or $F$. walujewii. Meanwhile, F. chuanxiensis and Zhejiang fritillary are both dominant products in the domestic medicinal materials market in China (Shan et al. 2017). Fritillaria is widely distributed with complex germplasm resources. People from various localities have different customs in the application of these materials. In addition, the prices of Fritillaria vary greatly based on the species and origins. Therefore, to ensure the efficacy and safety of this medical treatment, it is important to carry out research on the sources of this medicinal material.

The Bulbus $F$. thunbergii in the group of "eight herbal medicines of Zhejiang" is a famous Chinese medicinal material in Zhejiang Province (Yuan et al. 2010). It is of high quality and stable texture, and the associated products are sold in many places within and outside the province and further to countries and regions in Southeast Asia. This material is large with a shape of Yuanbao (gold or silver ingots with a Chinese style); thus, it is also called Yuanbao fritillary. Wild $F$. thunbergii is distributed in southern Jiangsu, northern Zhejiang, Hunan, etc. Large-scale cultivations occur in Ningbo, Pan'an of Zhejiang Province, and Nantong in Jiangsu Province, and it is also cultivated in other provinces, such as Hunan, Hubei, Sichuan, Anhui, and Henan. However, besides the cultivation bases, majority of medicinal materials are purchased from individual farmers. Problems, such as unclear sources and mixed varieties, often occur. To ascertain the authenticity of medicinal materials, to establish quality standards for the varieties, and to guide
Good Agricultural Practices (GAP) production, we conducted a chemical fingerprint study of $F$. thunbergii.

Thus far, more than 130 compounds have been isolated and identified from Fritillaria plants. These compounds are mainly alkaloids, most of which are isosteroidal alkaloids, and only a few are steroidal alkaloids or other types of alkaloids (Temel et al. 2015; Smolskait et al. 2015; Wang et al. 2018). Based on the heterocyclic structures of these compounds, isosteroid alkaloids from Fritillaria can be divided into the cevanine, jervine, and veratramine groups (Lyu et al. 2015; Ruan et al. 2016; Wang et al. 2018). There are also 15 steroidal alkaloids and one other alkaloid (Arshadi et al. 2012; Temel et al. 2015).

Currently, methods for the identification of Fritillaria include high-performance liquid fingerprint chromatography (HLPC), thin-layer chromatography, infrared, and ultraviolet spectroscopies, etc. Recently, there have been many research studies on HPLC fingerprinting. Zhang et al. (2015) established a fingerprint analytical method for $F$. hupehensis using HPLC-ELSD using the experimental details as follows: the column was a Hypersil ODS $(250 \times 4.6 \mathrm{~mm})$, mobile phase was methanol (containing $0.05 \%$ triethylamine)-water for gradient elution, flow rate was $1.0 \mathrm{~mL} / \mathrm{min}$, and detector was an ELSD. Li et al. (2006) examined 11 Fritillaria samples using HLPC according to the following details: XDP-C18 $(4.6 \times 250$ $\mathrm{mm}$ ) as the chromatographic column, the mobile phase was methanol-water in the ratio of 70:30 (v/v) containing 7.5 $\mathrm{mmol} / \mathrm{L}$ sodium dodecyl sulfate (SDS) with the $\mathrm{pH}$ at $4.5 \pm$ 0.1 , and the detection wavelength was $215 \mathrm{~nm}$. Based on the retention time and relative peak area of different chemical components in the sample, chemometric methods were applied to calculate and cluster the important parameters used to describe the degree of separation and quality of two adjacent chromatographic peaks. The results revealed the consistency between the cluster analysis and the botanical classification. This method can be used for the identification of Fritillaria, and it aided the establishment of the characteristic fingerprints of Fritillaria. Chen et al. (2013) used HPLC-differential thermal analysis (DTA) to determine the quality of $F$. thunbergii, $F$. thunbergii var. chekiangensis, $F$. hupehensis, and $F$. anhuiensis obtained under the same ecological environments with Fritillaria origins and different processing methods. These studies showed that Fritillaria samples processed by traditional methods contained the same ingredients and can be mixed, whereas samples fumigated with sulfur were different owing to the presence of sulfur dioxide; additionally, the Fritillaria dried with lime was stable (Mitra et al. 2009). Wang et al. (2017) reported that the alkaloids of Fritillaria from Jilin indicated significant difference in the HPLC spectra of extracts using different solvents and different methods. Lim et al. (2018) established an HPLC-ELSD method for the detection of Fritillaria alkaloids and glucoalkaloid to provide an accurate and concise approach for quality control of Fritillaria medicinal materials. 
In addition to HPLC fingerprinting, many researchers also use thin-layer chromatography to identify Fritillaria medicinal materials. Wu et al. (2010) applied thin-layer chromatography to compare $F$. ussuriensis of different origins and harvesting periods with different varieties of Fritillaria and counterfeits, and this method provided a reliable basis for the identification of the medicinal materials of $F$. ussuriensis. Li et al. (2006) used microscopic examination and TLC to identify $F$. pallidiflora with $F$. walujewii as reference substances. Rashid et al. (2015) identified and differentiated fresh tubers of $F$. thunbergii and its adulterant with TLC and ultraviolet spectral identification to provide a basis for further identification. Huang et al. (2005) analyzed the total saponins from various Fritillaria medicinal materials using thin-layer chromatography to distinguish $F$. chuanxiensis from other fritillary materials.

There have been also many studies on the identification of Fritillaria using spectroscopic techniques. Lee et al. (2015) combined near-infrared diffuse reflectance spectroscopy and principal component analysis (PCA) to show that this approach can identify Fritillaria and its related species, being capable of clustering and identification. Mitra et al. (2009) established a method using near-infrared diffuse reflectance spectroscopy for the identification of Fritillaria and its counterfeits, providing a new method for the identification and analysis of Fritillaria and other TCM. Smolskait et al. (2015) used FTIR spectroscopy and cluster analysis to identify and analyze Fritillaria medicinal materials and their counterfeits. $\mathrm{Li}$ et al. (2014) applied FTIR spectroscopy to directly determine the FTIR spectra of TCM, which provided a direct, quick, and accurate measurement of $F$. chuanxiensis, $F$. thunbergii, and $F$. thunbergii var. chekiangensis. Majo et al. (2011) used UV spectrophotometry to measure and compare the UV spectra of anhydrous ethanol extracts of $F$. walujewii and four other types of Fritillaria samples. The results revealed that $F$. walujewii showed consistent differences from the other four types of Fritillaria.

Although there are many studies on the chemistry and fingerprints of $F$. thunbergii, the fingerprints of medicinal materials produced in Pan'an County, which is the main production site of medicinal materials in Zhejiang Province, have not been studied previously. This study focused on the chemical fingerprinting of medicinal materials of $F$. thunbergii produced in Pan'an to establish quality control standards for the medicinal materials produced in Pan'an County and to guide GAP production.

\section{Materials and Methods}

\section{Test materials}

The test materials were $F$. thunbergii samples collected from Pan'an, Ningbo of Zhejiang Province and Nantong of Jiangsu Province, $F$. chuanxiensis from Maoxian, Sichuan
Province, and $F$. ussuriensis from Jilin Province during the spring and summer time of 2018 2019 (Table 1). Among them, there are two varieties of $F$. chuanxiensis: one has a shorter growth period and a smaller bulb, and the other has a longer growth period and a larger bulb.

\section{Preparation of test solutions}

According to the Chinese Pharmacopoeia (2020 edition), the extraction method of $F$. thunbergii was slightly modified to prepare the test solution. A $2 \mathrm{~g}$ of the test powder were accurately weighed, placed in an Erlenmeyer flask, and immersed in $4 \mathrm{~mL}$ of concentrated ammonia solution for 1 h. Forty $\mathrm{mL}$ of a chloroform-methanol (4:1) solvent was added, weighed, mixed well, and the flask was placed in a $40{ }^{\circ} \mathrm{C}$ water bath for $30 \mathrm{~min}$ and filtered. The filtrate was evaporated to dryness, and the residue was dissolved in methanol (chromatography grade) and transferred to a 10 $\mathrm{mL}$ volumetric flask. Methanol was added to make up the final volume, and the solution was filtered through a 0.45 $\mu \mathrm{m}$ filter membrane.

\section{Preparation of the reference solution}

Peimine and peiminine standards were accurately weighed and dissolved in methanol during ultrasonication to make a $0.1 \mathrm{mg} / \mathrm{mL}$ reference solution.

\section{Setup of chromatographic conditions}

As described by Wei et al. (2017), methanol-water (0.03\% triethylamine) was used as the mobile phase for gradient elution. The experimental results were promising. The following gradient elution procedure was set up through repeated experiments: flow rate of $1 \mathrm{~mL} / \mathrm{min}$, injection volume of $5 \mu \mathrm{L}$. The ELSD detection parameters were as follows: drift tube temperature of $35^{\circ} \mathrm{C}$ and carrier gas flow rate of $2.2 \mathrm{~L} / \mathrm{min}$.

\section{Data analysis}

Similarity analysis of fingerprints: Fingerprints were exported in AIA format from the Agilent chromatographic workstation, and imported into the 'Chinese Herbal Medicine Chromatographic Fingerprint Similarity Evaluation System' (promulgated by the National Pharmacopoeia Commission, Version A) software. Multiple operation steps were performed to obtain the analytical results, including trimming of the first $5 \mathrm{~min}$ of the chromatograms, baseline adjustment, multi-point correction, automatic matching, and similarity analysis after generating the control spectra.

Cluster analysis: Peaks in the fingerprint spectra were automatically integrated using the Agilent chromatographic workstation to obtain the area of each peak and to calculate the relative peak area by dividing each peak by the area of 
He et al. / Intl J Agric Biol, Vol 25, No 1, 2021

Table 1: Sample of regular table design Details of plant materials collected from different regions

\begin{tabular}{|c|c|c|c|}
\hline No. & Name & Origin & Time \\
\hline$\overline{\mathrm{S} 1}$ & Fritillaria cirrhosa (small) & Songpinggou, Maoxian County, Sichu & Jun. 2018 \\
\hline $\mathrm{S} 2$ & F. cirrhosa (large) & Songpinggou, Maoxian County, Sichu & Jun. 2018 \\
\hline S3 & F. thunbergii var. chekiangensis & Yanshang village, Pan'an, Zhejiang & Jun. 2018 \\
\hline $\mathrm{S} 4$ & F. ussuriensis & Huinan County, Tonghua City, Jilin Province & Aug. 2018 \\
\hline 55 & F.anhuiensis & Cixia village, Pan'an, Zhejiang & Jun. 2018 \\
\hline S6 & F.anhuiensis & Yanshang village, Pan'an, Zhejiang & Jun. 2018 \\
\hline S7 & F. thunbergii & Zhangzhishan, Nantong, Jiangsu & Jun. 2018 \\
\hline S8 & F. thunbergii (many-seeded) & Houtang village, Pan'an, Zhejiang & Jun. 2018 \\
\hline S9 & F. thunbergii & Zhangshui, Ningbo, Zhejiang & Jun. 2018 \\
\hline $\mathrm{S} 10$ & F. thunbergii & Cixia village, Pan'an, Zhejiang & Jun. 2018 \\
\hline S11 & F. thunbergii & Houtang village, Pan'an, Zhejiang & Jun. 2018 \\
\hline $\mathrm{S} 12$ & F. thunbergii & Pantanlengshui, Pan'an, Zhejiang & Jun. 2018 \\
\hline $\mathrm{S} 13$ & F. thunbergii & Yangzhai village, Pan'an, Zhejiang & Jun. 2018 \\
\hline
\end{tabular}

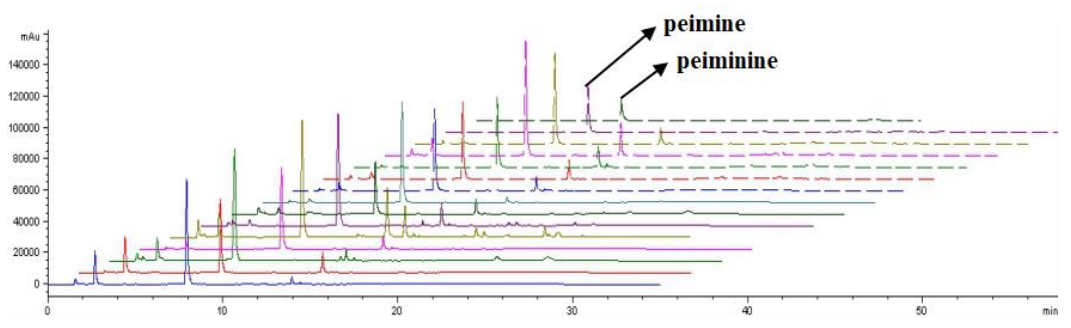

Fig. 1: HPLC-ELSD fingerprints of 13 Fritillaria samples

the corresponding peaks of peimine and peiminine. An absent peak was assigned a peak area of 0 . The relative peak area was used as the basis for clustering analysis. The cluster analysis function in the multivariate analysis menu in the DPS system was used to standardize the original data, based on Euclidean distance measurement (Saurabh et al. 2017) and shortest-distance clustering, DPS V8.01.

Principal component analysis (PCA): The relative peak area of each peak in the fingerprints was obtained in the same way as the cluster analysis. Based on the relative peak area, the PCA analysis function, which is an unsupervised multivariate data analysis approach, in the multivariate analysis menu in the DPS V8.01 was used to analyze the original data to obtain the PCA chart.

Calibration of chromatographic peaks in the HPLCELSD fingerprints: A $5 \mu \mathrm{L}$ of the standard solutions of peimine and peiminine were injected into a HPLC and measured according to the determined chromatographic conditions to produce the chromatograms (Fig. 1).

\section{Investigation of fingerprinting methodology}

After determination of the extraction and chromatographic separation conditions for the samples, we conducted a methodological investigation on the effectiveness of the entire method. Investigation indicators were selected from the following three aspects: the precision of the instruments, stability of the samples, and reproducibility of the method. Details are described as follows.

Precision test: A test solution of Fritillaria sample 6 was precisely injected at a volume of $5 \mu \mathrm{L}$ five consecutive times. The chromatogram was recorded, and the relative retention time, relative peak area, and RSD of each peak were calculated. The results showed that the RSD\% values of the relative retention time of the common peaks were between $0.18 \%$ and $3.01 \%$, and the RSD\% values of the relative peak area were between $2.1 \%$ and $3.46 \%$. ELSD detection has lower precision than UV detection, but it basically meets the technical requirements of fingerprints, controlled within $5 \%$.

Stability test: A test solution of Fritillaria sample 5 was precisely injected at a volume of $5 \mu \mathrm{L}$ and at intervals of every $2,4,6,12$, and $24 \mathrm{~h}$. The results revealed that the $\mathrm{RSD} \%$ values of the relative retention time of the common peaks were from 1.6 to 2.84 , which were all less than $3 \%$. The RSD\% values of the relative peak area were between $1.9 \%$ and $4.23 \%$, which indicated that the tested product was stable within $24 \mathrm{~h}$.

Repeatability test: Five replicates of Fritillaria sample 6 were prepared as the test solution according to the determined method. The solutions were injected separately, and the chromatograms were recorded. The results showed that the RSD\% values of the relative retention time of the common peaks were between $0.97 \%$ and $1.35 \%$, which were all less than $3 \%$. RSD\% values of the relative peak area were between $2.74 \%$ and $4.26 \%$. Fig. 1 shows that the retention times of peimine and peiminine standards were 8.102 and $8.252 \mathrm{~min}$, respectively, and the time difference between the two was only $0.15 \mathrm{~min}$. The retention times of peimine and peiminine in the test solutions were almost the same, showing a large peak at approximately $8 \mathrm{~min}$ in the fingerprints. 


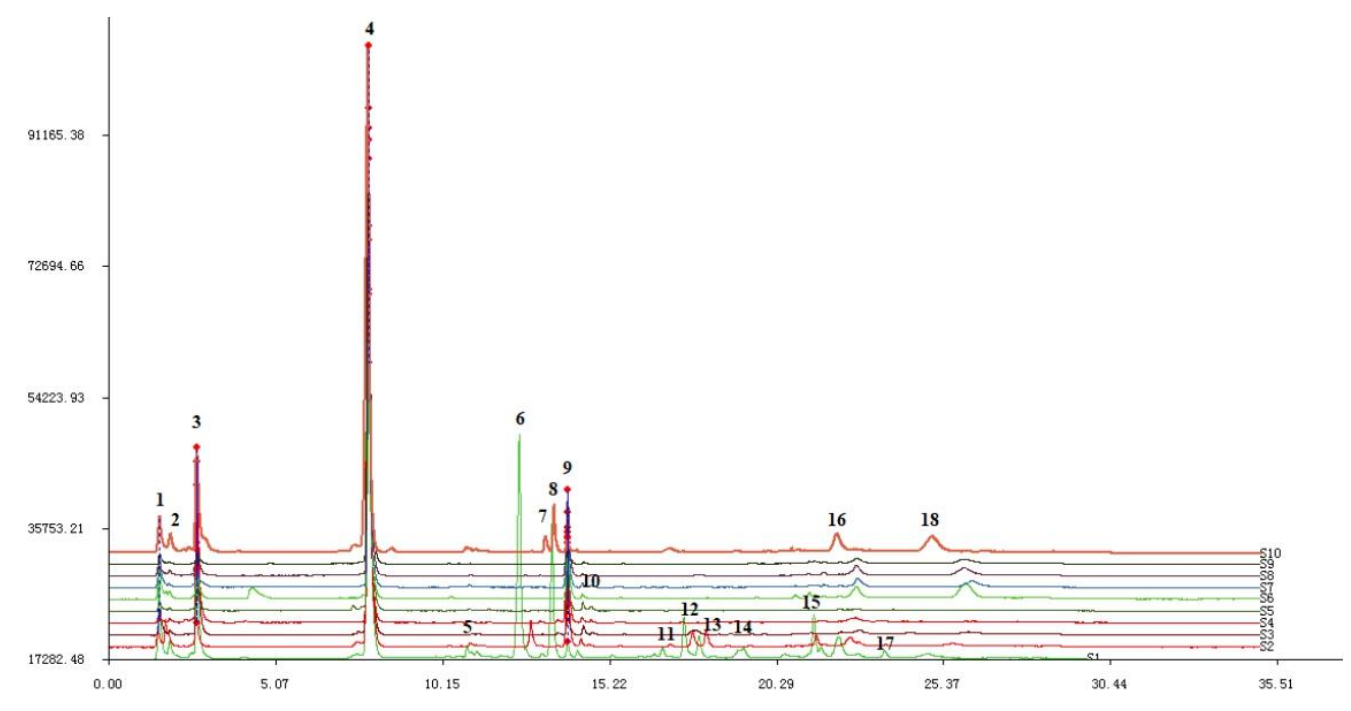

Fig. 2: Compare of different Fritillaria species (after calibration)

\section{Results}

\section{Analysis and comparison of HPLC fingerprints of different species of Fritillaria}

In this study, the HPLC spectra of $F$. thunbergii, $F$. thunbergii var. chekiangensis, $F$. anhuiensis, $F$. chuanxiensis, and F. ussuriensis were compared. Fig. 2 shows that the spectra of different species of Fritillaria are quite different. The chromatograms were imported into the "Chinese Herbal Medicine Chromatographic Fingerprint Similarity Evaluation System" in AIA format with multipoint correction. $F$. anhuiensis had the highest number of peaks. Although $F$. thunbergii var. chekiangensis is a variant of $F$. thunbergii, its chemical composition was significantly different from $F$. thunbergii with an extra peak (No. 7) compared to other Fritillaria, and the retention time of each peak was quite different from that of other samples. The HPLC spectrum of multi-seed $F$. thunbergii was similar to that of $F$. thunbergii, but its content is not as high as that of $F$. thunbergii. Number 10 peak was present in the spectra of $F$. ussuriensis, $F$. chuanxiensis, and F. anhuiensis but not in the spectra of $F$. thunbergii and $F$. thunbergii var. chekiangensis.

\section{HPLC-ELSD fingerprint similarity of 13 batches of Fritillaria}

Similarity analysis was carried out using the method specified previously. The results showed that the similarities among the $F$. thunbergii samples were all above 0.90 . The similarities among $F$. chuanxiensis (large), $F$. anhuiensis and $F$. thunbergii were between 0.80 and 0.90 , indicating that they can be distinguished. However, the similarity between $F$. ussuriensis and $F$. thunbergii was also above
0.90, implying the difficulty in distinguishing these two species.

\section{Cluster analysis of 13 batches of Fritillaria}

Different Fritillaria samples were considered as one entity. By comparing the properties and characteristics of the relative peak area change among the different samples, i.e., the areas of all peaks were divided by the peak area of the No. 4 peak, the relative peak areas of the 13 Fritillaria samples were systematically clustered by the DPS system (Table 2). The Euclidean distance was used for the measurement, and clustering was done using the minimum distance method (Fig. 3).

Results showed that the $F$. anhuiensis product grown in Cixia village of Pan'an showed the most significant difference from other Fritillaria species, followed by $F$. chuanxiensis with a longer growth period (Fig. 4). F. thunbergii samples grown in Nantong of Jiangsu were not clustered together with the samples of the same species grown in Zhejiang, indicating a difference between the chemical compositions of the $F$. thunbergii samples from these two sources, which may be influenced by the growing environment. Although $F$. thunbergii var. chekiangensis is a variety of $F$. thunbergii, its chemical composition was significantly different from that of $F$. thunbergii. The difference was evident in the fingerprints as follows: peaks 2 and 7 are absent from the spectrum of $F$. thunbergii, and they cluster with $F$. chuanxiensis, which has a short growth period. $F$. ussuriensis and the $F$. thunbergii produced in Cixia and Yangzhai clustered together. However, according to the HPLC spectra, $F$. anhuiensis and $F$. thunbergii can be easily distinguished. It is likely that the source of $F$. anhuiensis was different. The fingerprints of $F$. ussuriensis and $F$. thunbergii were very similar, and $F$. ussuriensis 
He et al. / Intl J Agric Biol, Vol 25, No 1, 2021

Table 2: Similarity of HPLC fingerprints from 13 Fritillaria sample

\begin{tabular}{|c|c|c|c|c|c|c|c|c|c|c|c|c|c|}
\hline & $\mathrm{S} 1$ & $\mathrm{~S} 2$ & S3 & $\mathrm{S} 4$ & S5 & S6 & S7 & S8 & S9 & S10 & S11 & S12 & S13 \\
\hline $\mathrm{S} 1$ & & 0.856 & 0.95 & 0.965 & 0.872 & 0.963 & 0.902 & 0.979 & 0.942 & 0.974 & 0.927 & 0.977 & 0.979 \\
\hline S2 & 0.856 & & 0.862 & 0.928 & 0.816 & 0.891 & 0.914 & 0.878 & 0.931 & 0.892 & 0.893 & 0.891 & 0.893 \\
\hline S3 & 0.95 & 0.862 & & 0.97 & 0.908 & 0.972 & 0.903 & 0.975 & 0.944 & 0.956 & 0.933 & 0.954 & 0.967 \\
\hline S4 & 0.965 & 0.928 & 0.970 & & 0.902 & 0.992 & 0.945 & 0.99 & 0.984 & 0.983 & 0.966 & 0.981 & 0.99 \\
\hline S5 & 0.872 & 0.816 & 0.908 & 0.902 & & 0.921 & 0.857 & 0.894 & 0.893 & 0.900 & 0.886 & 0.9 & 0.903 \\
\hline S6 & 0.963 & 0.891 & 0.972 & 0.992 & 0.921 & & 0.936 & 0.988 & 0.978 & 0.985 & 0.966 & 0.984 & 0.991 \\
\hline S7 & 0.902 & 0.914 & 0.903 & 0.945 & 0.857 & 0.936 & & 0.931 & 0.959 & 0.958 & 0.976 & 0.936 & 0.95 \\
\hline S8 & 0.979 & 0.878 & 0.975 & 0.99 & 0.894 & 0.988 & 0.931 & & 0.965 & 0.982 & 0.957 & 0.975 & 0.992 \\
\hline S9 & 0.942 & 0.931 & 0.944 & 0.984 & 0.893 & 0.978 & 0.959 & 0.965 & & 0.981 & 0.97 & 0.978 & 0.98 \\
\hline S10 & 0.974 & 0.892 & 0.956 & 0.983 & 0.900 & 0.985 & 0.958 & 0.982 & 0.981 & & 0.984 & 0.994 & 0.998 \\
\hline S11 & 0.927 & 0.893 & 0.933 & 0.966 & 0.886 & 0.966 & 0.976 & 0.957 & 0.97 & 0.984 & & 0.967 & 0.978 \\
\hline $\mathrm{S} 12$ & 0.977 & 0.891 & 0.954 & 0.981 & 0.900 & 0.984 & 0.936 & 0.975 & 0.978 & 0.994 & 0.967 & & 0.992 \\
\hline S13 & 0.979 & 0.893 & 0.967 & 0.99 & 0.903 & 0.991 & 0.95 & 0.992 & 0.98 & 0.998 & 0.978 & 0.992 & \\
\hline
\end{tabular}

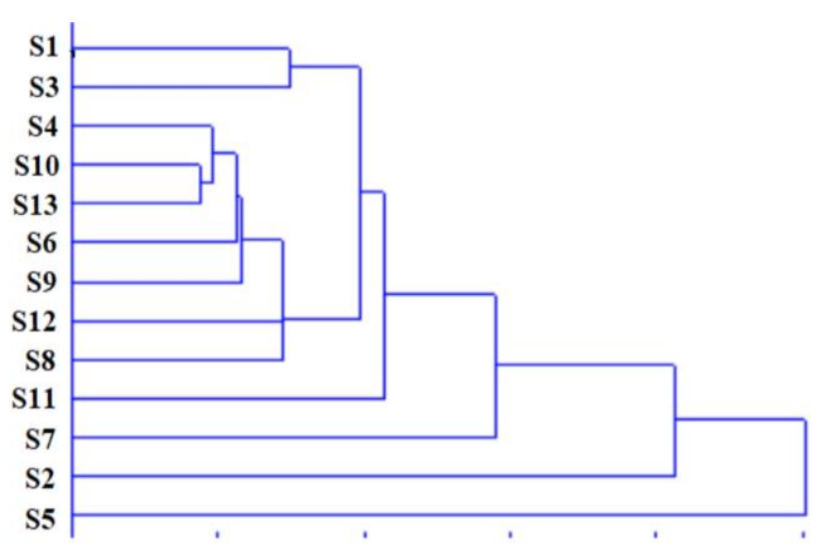

Fig. 3: Result of cluster analysis for Fritillaria species

showed an extra peak (No. 9) compared with the spectrum of $F$. thunbergii.

\section{PCA of 13 batches of Fritillaria}

In addition to the cluster analysis of the HPLC fingerprints of Fritillaria, we also applied PCA to analyze the data of all Fritillaria samples (Fig. 5). It was concluded that samples $\mathrm{S} 7$ and S5 were quite different from other samples; $F$. thunbergii samples produced in Nantong of Jiangsu and $F$. anhuiensis samples produced in Cixia of Pan'an, showed different HPLC fingerprints from other Fritillaria samples. This indicated that there was a difference in the composition or content between the $F$. thunbergii samples produced in Jiangsu and Zhejiang Province, which further confirmed the authenticity of these medicinal materials. Almost all other samples clustered together, and they could not be compared. However, the PCA results can be combined with the cluster analysis results to reveal information as follows. Three samples with the greatest differences were $F$. anhuiensis from Cixia Village of Pan'an, F. thunbergii from Nantong of Jiangsu, and $F$. chuanxiensis with a longer growth period. $\mathrm{S} 2, \mathrm{~S} 3, \mathrm{~S} 4$ and S6 were distributed in the sample region with all other $F$. thunbergii samples, but were not distinguished in this analysis.

\section{Discussion}

The HPLC fingerprints of different species of Fritillaria were analyzed by HPLC-ELSD and further compared. The results showed that the greatest difference was between $F$. anhuiensis from Cixia Village of Pan'an and other Fritillaria samples. Under the same extraction and detection conditions, $F$. anhuiensis have more fingerprint peaks than those of $F$. thunbergii, $F$. chuanxiensis and $F$. ussuriensis. $F$. anhuiensis samples are clustered together with $F$. thunbergii in the clustering charts, but their HPLC spectra indicate that some peaks of the two types of $F$. anhuiensis samples are consistent, such as peak No. 6, 12, 13, and 15, all of which are unique to $F$. anhuiensis (Fig. 3). Further investigation is needed to determine the causes of this difference. One possible reason is that local farmers introduced seeds from different sources. The other is that these two $F$. anhuiensis samples may belong to different species. In addition, $F$. anhuiensis is just a vague collective term for Fritillaria species in Anhui. There are many specific varieties, such as Anhui Fritillaria, Wanjiang Fritillaria, Wannan Fritillaria, and Wanxi Fritillaria (Paz et al. 2015). Second, F. chuanxiensis with a longer growth period is isolated, whereas $F$. chuanxiensis with a shorter growth period was gathered first with $F$. thunbergii var. chekiangensis and then with $F$. thunbergii, indicating that the chemical composition of Fritillaria is a dynamic accumulation process. The chemical composition of Fritillaria with different growth years may not be identical, which provides a basis for the harvest time limits of medicinal materials. F. thunbergii var. chekiangensis is a variety of $F$. thunbergii. It is mainly produced in Pan'an, Zhejiang. It has a smaller size, similar to $F$. chuanxiensis. It has been used to replace $F$. chuanxiensis by the public. However, its chemical composition is closer to that of $F$. thunbergii with some differences. Zhao et al. (2014) analyzed it chemical composition and confirmed that it is a variety of $F$. thunbergii. It is quite different from $F$. chuanxiensis and cannot replace it. Of the $F$. thunbergii samples, there was no obvious difference between the $F$. thunbergii collected in Zhejiang Province (Pan'an and Ningbo), but the $F$. thunbergii from Nantong, Jiangsu was 
isolated separately, indicating that there were differences in F. thunbergii from different regions. This may have been caused by the growing environment, and it further confirmed the authenticity of the medicinal materials.

In this study, $F$. ussuriensis and $F$. thunbergii were clustered together. A review of relevant studies showed that the main alkaloid components in $F$. ussuriensis are pingpeimine A and pingpeimine B (Sun et al. 2013), which have similar chemical structures as peimine and peiminin. Thus, they may have retention times that are very close to those of $F$. thunbergii in the HPLC fingerprints. $F$. ussuriensis also contains peimine at a level of 0.002 $0.003 \%$ (Chen et al. 2011), which may coelute with pingpeimine $\mathrm{A}$ and pingpeimine $\mathrm{B}$ to form a huge peak at approximately $8 \mathrm{~min}$. The above reasons caused their fingerprints to be very similar in this study and not distinguishable.

Based on the HPLC-ELSD fingerprints of 13 batches of Fritillaria, five types, i.e., $F$. thunbergii, $F$. thunbergii var. chekiangensis, $F$. chuanxiensis, $F$. anhuiensis and $F$. ussuriensis, were compared to obtain preliminary results. However, there exist some problems and issues. 1) there were only 13 batches of samples with only eight batches of $F$. thunbergii samples (seven taken within the province and one taken outside the province). To establish the HPLC fingerprints of $F$. thunbergii from Zhejiang Province, at least 10 batches of samples from places within the province were required. Thus, the number of samples needs to be increased; 2) information on the samples was partially missing, for example, the purchased $F$. anhuiensis sample did not have a specific species name and 3) the HPLC detection conditions need to be further optimized.

\section{Conclusion}

HPLC-ELSD fingerprints could well distinguish $F$. thunbergii grown in Pan'an, and $F$. thunbergii var. chekiangensis, $F$. anhuiensis, $F$. thunbergii, F. chuanxiensis, and $F$. ussuriensis samples from places outside Zhejiang Province, indicating that the environment has an important influence on the composition of medicinal materials, which confirmed the authenticity of the medicinal materials. In this study, only the fingerprints of the alkaloids of Fritillaria were studied. The non-alkaloids were excluded because many of the components in Fritillaria are similar in structure, only differing in some of the substituent groups. This makes it difficult to distinguish these components in the HPLC spectra, and more investigation is needed to optimize the HPLC separation conditions.

\section{Acknowledgement}

This research was supported by National Natural Science Foundation of China (31600257 and 31800187) and Natural Science Foundation of Zhejiang Province (LY18C030003).

\section{Author Contributions}

ZJ conceived the idea of work; QM conducted formal analysis; RH investigated the materials; $\mathrm{RH}$ and QM wrote the original draft; $\mathrm{YL}$ and JZ reviewed and edit the draft. All authors have read and agreed to the published version of the manuscript.

\section{References}

Arshadi M, AJ Hunt, JH Clark (2012). Supercritical fluid extraction (SFE) as an effective tool in reducing auto-oxidation of dried pine sawdust for power generation. RSC Adv 2:1806-1809

Chen LH, LL Liu, WF Zhu, HM Zhang, ZH Yan, HN Liu (2011). Comparative pharmacokinetic studies of peimine and peiminine in rat plasma by LC-MS-MS after oral administration of Fritillaria thunbergii Miq. and Fritillaria thunbergii Miq.Glycyrrhiza uralensis Fisch. couple extract. Pharmazie 66:684 689

Chen LH, HM Zhang, ZY Guan (2013). Sex dependent pharmacokinetics, tissue distribution and excretion of peimine and peiminine in rats assessed by liquid chromatography-tandem mass spectrometry. $J$ Ethnopharmacol 145:77-84

Chinese Pharmacopoeia Commission. The Pharmacopoeia of the People's Republic of China (2020 edn). Pharmaceutical Science and Technology Press, 2020, Beijing, China

Gao Y, B Ma, Q Liu, YY Wang (2011). Methodological study and establishment of the diagnostic scale for TCM syndrome of ischemic stroke. J Tradit Chin Med 24:1-12

Huang D, B Ou, RL Prior (2005). The chemistry behind antioxidant capacity assays. J Agric Food Chem 53:1841-1856

Lee B, EY Kim, JH Kim, JH Min, DW Jeong, JY Jun, CY Cho, Y Sohn, HS Jung (2015). Antiallergic effects of peiminine through the regulation of inflammatory mediators in HMC-1 cells. Immunopharmacol Immunotoxicol 37:351-358

Li DQ, J Zhao, SP Lia (2014). High-performance liquid chromatography coupled with post-columndual-bioactivity assay for simultaneous screening of xanthine oxidaseinhibitors and free radical scavengers from complex mixture. J Chromatogr A 1345:50-56

Li HJ, Y Jiang, P Li (2006). Chemistry, bioactivity and geographical diversity of steroidal alkaloids from the Liliaceae family. Nat Prod Rep 23:735-752

Lim JM, B Lee, JH Min, EY Kim, JH Kim, S Hong, JJ Kim, Y Sohn, HS Jung (2018). Effect of peiminine on DNCB-induced atopic dermatitis by inhibiting inflflammatory cytokine expression in vivo and in vitro. Intl Immunopharmacol 56:135-142

Liu HJ, TH Li, LX Chen, S Zhan, ML Pan, ZG Ma, CH Li, Z Zhang (2016). To set up a logistic regression prediction model for hepatotoxicity of Chinese Herbal Medicines based on Traditional Chinese Medicine theory. Evid-Based Compl Alt 2016; Article 7273940

Lyu Q, F Tou, H Su, X Wu, X Chen, Z Zheng (2015). The natural product peiminine represses colorectal carcinoma tumor growth by inducing autophagic cell death. Biochem Biophys Res Commun 462:38-45

Majo DD, LL Neve, ML Guardia, A Casuccio, M Giammanco (2011). The influence of two different $\mathrm{pH}$ levels on the antioxidant properties of flavonols, flavan-3-ols, phenolic acids and aldehyde compounds analysed in synthetic wine and in a phosphate buffer. J Food Compos Anal 24:265-269

Mitra P, HS Ramaswamy, KS Chang (2009). Pumpkin (Cucurbita maxima) seed oil extraction using supercritical carbon and physicochemical properties of the oil. J Food Eng 95:208-213

Rashid JIA, NA Yusof, J Abdullah, U Hashim, R Hajian (2015). Surface modifications to boost sensitivities of electrochemical biosensors using gold nanoparticles/silicon nanowires and response surface methodology approach. J Mater Sci 51:1083-1097 
Paz M, P Gúllon, MF Barroso, AP Carvalho, VF Domingues, AM Gomes, H Becker, E Longhinotti, C Delerue-Matos (2015). Brazilian fruit pulps as functional foods and additives: Evaluation of bioactive compounds. Food Chem 172:462-468

Qu JH, B Liu, ZQ Zhang, DP Liu (2014). Capturing the opportunities to initiate an in-depth integration of traditional Chinese medicine and western medicine. Front Med 8; Article 268

Ruan X, L Yang, WX Cui, MX Zhang, ZH Li, B Liu, Q Wang (2016). Optimization of supercritical fluid extraction of total alkaloids, peimisine, peimine and peiminine from the bulb of Fritillaria thunbergii Miq, and evaluation of antioxidant activities of the extracts. Material 9; Article 524

Saurabh KS, Edmond YML, XS Qin (2017) Cluster analysis of monthly precipitation over the western maritime continent under climate change. Climate 5; Article 84

Shan YG, ZM Yu, YQ Fu (2017). Quality comparison on Zhejiang Fritillary before and after its migration to Qingyuan and its fingerprint. Chin Arch Tradit Chin Med 5:1257-1260

Smolskait L, PR Venskutonis, T Talou (2015). Comprehensive evaluation of antioxidant and antimicrobial properties of different mushroom species. LWT-Food Sci Technol 60:462-471

Sun YG, YF Du, K Yang, L Chang, L Cao, YP Ren, Q Sun, Q Wang, LT Zhang,PTLv (2013). Acomparative study on the pharmacokinetics of a traditional Chinese herbal preparation with the single herb extracts in rats by LC-MS/MS method. J Pharm Biomed Anal 81-82:34-43

Temel E, C Alasalvar, H Gökçe, A Güder, Ç Albayrak, YB Alpaslan, G Alpaslan, N Dilek (2015). DFT calculations, spectroscopy and antioxidant activity studies on (E)-2-nitro-4-[(phenylimino) methyl] phenol. Spectrochim Acta A 136:534-546
Wang ZB, F Cao, YJ Chen, ZQ Tang, ZY Wang (2018). Simultaneous determination and pharmacokinetics of peimine and peiminine in beagle dog plasma by UPLC-MS/MS after the oral administration of Fritillariae ussuriensis Maxim and Fritillariae thunbergii Miq powder. Molecules 23:1573-1579

Wang Z, W Zhu, M Gao, C Wu (2017). Simultaneous determination of cucurbitacin $\mathrm{B}$ and cucurbitacin $\mathrm{E}$ in rat plasma by UHPLC-MS/MS: A pharmacokinetics study after oral administration of cucurbitacin tablets. J Chromatogr B 63:1065-1066

Wei BB, ZX Chen, MY Liu, MJ Wei (2017). Development of a UPLCMS/MS method for simultaneous determination of six flavonoids in rat plasma after administration of Maydis stigma extract and its application to a comparative pharmacokinetic study in normal and diabetic rats. Molecules 22:1267

Wu XD, JJ Chen, YJ Pan (2010). Simultaneous determination of peimine and peiminine in rat plasma by LC-ESI-MS employing solid-phase extraction. Biomed Chromatogr 24:902-907

Yuan XF, J Xu, H Chai, HR Lin, YQ Yang, XD Wo, JY Shi (2010). Differences of rhizo-bacterial diversity and the content of peimine and peiminine of Fritillaria thunbergii among different habits. $J \mathrm{Med}$ Plants Res 4:465-470

Zhang B, ZY Deng, DD Ramdath, Y Tang, PX Chen, RH Liu, Q Liu, R Tsao (2015). Phenolic profiles of 20 Canadian lentil cultivars and their contribution to antioxidant activity and inhibitory effects on $\alpha$ glucosidase and pancreatic lipase. Food Chem 172:862-872

Zhao MM, WJ Ding, S Wang, M Gao, S Fu, J Zhang, T Li, Y Wu, Q Wang (2014). Simultaneous determination of five constituents in qinpijiegu capsule by high-performance liquid chromatography coupled with tandem mass spectrometry. J Chromatogr Sci 53:274-279 